\title{
La eficiencia del impuesto a la exportación del café en El Salvador*
}

\author{
Oscar Edgardo Melhado
}

\section{Introducción}

El Salvador, Malasia, Sri Lanka, Ruanda y otros palses africanos son las naciones con mayor dependencia de los impuestos a la exportación. Esto se debe a la estructura económica particular de esos palses. Siendo el café para El Salvador una actividad económica importante, representa una base de impuestos adecuada. El Impuesto a la exportación del café ha sido un componente importante en las recaudaciones fiscales en EI Salvador. El impuesto representa una transferencia de recursos de los grupos cafetaleros al gobierno.

Al proponernos un análisis del impuesto a la exportación del café, enfocamos las distorsiones causadas por el gravamen. Esto implica que el impuesto genera un costo social. Este costo social, o carga excesiva, es minimizado cuando el comportamiento de los agentes económicos no es modificado por la imposición del impuesto. Para emprender nuestro análisis de eficiencia se requiere un estudio del café por el lado de la oferta. Caracterizando la oferta de café podernos determinar los costos en que la sociedad incurre por la utilización del impuesto a la exportación.

- Este articulos es un resumen de uno de los capltulos de la tesis doctoral del autor, titulada Taxation of Coffee Exports and the Behavior of Coffee Prices in El Salvador. La tesis presentada on Boston University en diciembre de 1990. 
En este artículo buscaremos clarificar las ineficiencias que acompanan al impuesto a la exportación de café. El análisis utiliza un modeto de producción y deriva el cálculo de la carga excesiva en equilibrio parcial. Además, en una extensión, calcula la carga excesiva tomando en consideración alteraciones en el tipo de cambio debidos a desequilibrio en la balanza de pagos. El trabajo se ha estructurado de la manera siguiente: en la sección II se formula algunas consideraciones acerca del impuesto a la exportación del café. En la sección III enfocamos los impuestos implicitos al café provenientes de otras fuentes. La sección IV describe y estima el modelo de producción, obteniendo la elasticidad precio de la oferta, para luego medir la carga excesiva en la sección V. La sección final trata sobre la carga excesiva en un modelo de balanza de pagos.

\section{Consideraclones prellmInares acerca del Impuesto a la exportación y a la exportación del café}

Existe equivalencia entre los impuestos a la exportación y los aranceles, asumiendo el ajuste adecuado en la balanza de pagos.' $\mathrm{El}$ impuesto óptimo a la exportación, sigue la misma regla que el arancel óptimo. En el caso de que el pais tenga poder monopólico en los mercados internacionales, el impuesto óptimo a la exportación es el inverso de la elasticidad precio de la demanda en el largo plazo. En el caso de El Salvador, esta regla no puede ser aplicada debido a que toma el precio intemacional como dado; de manera que no tiene influencia alguna en el precio internacional del café. En otras palabras, la carga del impuesto no puede ser traspasada a los consumidores en el mercado internacional.

A la misma conclusión se llega de la aplicación del teorema de producción eficiente de Diamond y Mirrlees (1971). La política óptima si el país tiene poder monopólico en el mercado internacional del café incluye impuestos al comercio internacional, de forma que las tasas marginales de transformación son equilibradas con las actividades de producción doméstica y el comercio exterior. ${ }^{2}$ El teorema de producción eficiente senala que no deben existir impuestos a los insumos. Si el comercio es considerado como otra actividad de producción, la implicación para una nación pequeńa en el mercado internacional -como el Salvador- es que los impuestos al comercio internacional deben ser evitados. Desde el punto de vista normativo, el poner impuestos a la exportación es considerado como una actividad no deseable.

El objetivo de cargar con impuestos a la exportación de café en EI Salvador es justificado en términos de los ingresos fiscales obtenidos. 
La recolección del impuesto a la exportación ha representado en los diez últimos anos un promedio de $23.86 \%$ del total de ingresos fiscales.

El impuesto a la exportación de café ha sido sujeto de discusión permanente, porque representa una transferencia de los cafetaleros al goblerno, y los grupos relacionados con la producción de café han tenido una influencia fundamental en la historia económica y social de El Salvador. La dependencia del café de esta nación, desde su introducción a finales del siglo pasado, ha sido notable. Este producto ha sido el principal recurso de divisas en este siglo, aunque en la historia reciente las remesas de inmigrantes en Estados Unidos y la ayuda intemacional están disputando esta supremacla. Las ganancias provenientes de este sector han sido invertidas en otras áreas. En el pasado las ganancias eran gravadas con un impuesto al ingreso; después de 1950, una tasa progresiva al precio internacional del café fue introducida a las exportaciones del café. ${ }^{3}$

La variedad de café producida en El Salvador está considerada en el grupo de "otros suaves". La política de exportación del café en este país ha estado relacionada con los sucesos en la diplomacia internacional del café. En los últimos 30 anos, las exportaciones han estado más influenciadas por los eventos en los acuerdos cafetaleros que por las fluctuaciones del precio internacional. De 1962 a 1971, el acuerdo del café, del cual El Salvador participó como miembro exportador, disminuyó la oferta internacional del grano mediante la asignación de cuotas de exportación. Los paises eran obligados a exportar la cuota permitida a los miembros importadores en un periodo dado de tiempo. ${ }^{5}$ Las exportaciones totales siempre excedieron la cuota.

El acuerdo era entre paises importadores y exportadores y fue orientado a traer estabilidad en los precios, entendida esta como la elevación de los precios a un nivel aceptable, más que a reducir la varianza de los mismos. Los precios fueron determinados por los mercados pero intervenidos por las regulaciones de las cuotas; las cuotas podian ser ajustadas en cualquier momento tomando en cuenta la fluctuación en los precios. Los objetivos generales del acuerdo eran:

a) Conseguir un balance entre oferta mundial y demanda.

b) Evitar fluctuaciones extremas en el nivel de la oferta.

c) Incentivar el consumo mundial de café.

Desacuerdos acerca de la distribución de las cuotas en 1972 condujeron a la suspensión de los controles y a una situación de libre mercado. Parece que EI Salvador aprovechó ese periodo para sacar sus 
inventarios acumulados de café. Esta es la razón por la cual el siguiente ano, aun con los precios altos, las exportaciones fueron bajas y fueron limitadas por la producción que cayó en ese año debido al clima adverso.

Durante el periodo de suspensión de las cuotas, algunas naciones latinoamericanas se unieron en un cartel con el objetivo de manipular el precio. Pero en 1975 una helada dańó severamente la producción brasilena y empujó los precios a niveles nunca antes alcanzados. La caida de la oferta produjo la eliminación de las restricciones. Pero el cartel latinoamericano intervino el mercado para mantener los precios altos. En 1977 y 1978 de nuevo, aún con los altos precios, las exportaciones fueron limitadas. En 1979, siguiendo una leve helada en Brasil y con la idea de que el sistema de cuotas sería reintroducido, todos los inventarios fueron vaciados. Este ano registra la más alta producción en El Salvador. ${ }^{6}$

Las cuotas fueron introducidas de nuevo en 1979 después de la liquidación del cartel latinoamericano conocido como "El Grupo de Bogotá". La cuota disenada para El Salvador para el periodo 1980-1986 se presenta en la Tabla 1. El nuevo acuerdo cafetalero se basó en el acuerdo de 1968, y reconoció la importancia de la estabilidad en precios para productores y consumidores. Además del mercado de paises importadores a donde la mayor parte de la cuota fue exportada, ha existido un mercado de no miembros en el cual el exceso de oferta ha sido vendido a precio de remate. El Salvador fue agresivo durante los anos 1983-1985 para vender su producción a países no miembros. Esta política no fue afortunada dado que los inventarios eran completamente bajos y asi no se pudo aprovechar los altos precios y la interrupción de la cuota en 1986 debido a la sequia en Brasil.

Tabla 1: Cuota para El Salvador \begin{tabular}{c} 
(miles de sacos) \\
\hline Ano
\end{tabular} Cuota
\begin{tabular}{|l|c|}
\hline $80 / 81$ & 2300 \\
$81 / 82$ & 2296 \\
$82 / 83$ & 2321 \\
$83 / 84$ & 2330 \\
$84 / 85$ & 2522 \\
$85 / 86$ & 2387 \\
\hline
\end{tabular}

Fuente: OIC. 
La cuota fue internumpida en 1986 y reintroducida después, pero no por mucho tiempo. El acuerdo del café fue liquidado en julio de 1989. Un ano antes, las condiciones climáticas en El Salvador danaron severamente la producción y las exportaciones no alcanzaron a llenar la cuota fijada.

La cuota global asignada a El Salvador en los acuerdos de 1976 y 1983 ha estado relacionada con la participación en votos en el acuerdo internacional del café. La cuota anual estuvo dividida en dos partes: una parte fija, correspondiente a no menos de $70 \%$ de la cuota global, y una parte variable de no menos de $30 \%$ de la cuota global. La parte variable dependió de la verificada disponibilidad de inventarios a ser evaluada anualmente. Esta es la razón porque la cuota cambiaba anualmente. El otro detalle importante fue el mercado de paises no miembros en los cuales se asignaba el exceso de exportaciones a precios más bajos.

\section{Tributación Implíclta en la exportación de café}

\section{a) Del tlpo de camblo y del sistema de aranceles}

Hay otros mecanismos diferentes al impuesto a la exportación que han afectado al sector cafetalero. Estos no constituyen sino distintas formas de tributación implícita a la exportación de café.

La primera forma de tributación implícita es a través de un tipo de cambio sobrevaluado. Los exportadores de café de esta manera reciben menos dinero doméstico que el valor real de las divisas que ellos ganan. Por otro lado, los importadores son subsidiados y pueden comprar bienes importados a un precio más bajo que su valor real. Por consiguiente los precios relativos entre el sector cafetalero y otras actividades son cambiados por esta política.

La tasa de cambio del mercado negro se puede considerar como un aproximado para el precio sombra del tipo de cambio.' Nótese en la figura 1 la diferencia entre las tasas oficiales y del mercado negro. Este margen es una aproximación de las ganancias adicionales negadas a los exportadores por un tipo de cambio sobrevaluado.

El segundo mecanismo por el cual el sector cafetalero ha sido impllcitamente cargado es a través de los aranceles y el sistema de exenciones. ${ }^{\ominus}$ El esquema de protección vuelve los precios relativos en contra del sector agroexportador. Un efecto similar es causado por la exención de aranceles adjudicadas a los insumos importados destinados al sector de exportaciones no tradicionales. 


\section{b) El monopollo gubernamental en la exportación del cafó}

El tercer mecanismo de tributación implicita ha sido a través de la agencia del gobierno que monopolizó la comercialización intemacional del café entre 1980 y 1989. Después de 1989, INCAFE es compotidor más en el mercado de exportaciones. Tiene un efecto de impuesto en el sentido de que el precio para los productores es reducido. Adicionalmente al impuesto y a las ganancias de INCAFE, los costos del mantenimiento burocrático de la institución fueron también descontados del precio dado a productores.

Además de consideraciones políticas, la principal causa que motivó la creación de INCAFE fue la acusación de que los exportadores estaban declarando menor valor de sus exportaciones para evadir el impuesto a la exportación. Una motivación adicional fue la reducción de la fuga de capitales proveniente de las ganancias del café.

\section{c) Preclos relatlvos en el sector agrícola}

Los precios relativos de la agricultura en términos de otros sectores como manufacturas, construcción, comercio, finanzas y servicios, han tenido una tendencia decreciente. La figura 2 muestra los deteriorados precios del sector agricola.' El principal componente en este deterioro es la situación adversa del café. Después del boom de los precios en 1977, los precios del café han disminuido en relación con otros sectores aún del mismo sector agricola. La figura 3 ilustra el caso del café. ${ }^{10}$

La exportación del café tiene todas las cargas anteriormente mencionadas. La conclusión es que el sesgo en contra del sector cafetalero está afectando los incentivos de producción.

\section{Modelo de producclón y Estimaclón}

No existe consenso acerca del método de evaluar los cambios en la oferta debido a fluctuaciones en los precios. Estimaciones de la elasticidad precio de la oferta de café son presentados en la Tabla 2."

Usaremos una versión del modelo de Nerbve, el cual incluye ajustes en el producto y una función del precio esperado.

En El Salvador, a pesar de la cuota, la expansión de la producción de café ha sido sensible al precio recibido por los productores. La proporción de la cuota (durante el acuerdo cafetalero de la OIC) estuvo sujeta a la producción y al área sembrada. El principal elemento para modelar la expansión de la producción es determinar como los precios esperados inducen a los productores a producir un determinado nivel de café. 
a) El modelo para estimar la funclón de oferta

Asumamos que la función de costos tiene la siguiente forma

$$
T C=(\gamma / 1+\gamma)(1 / a)^{1 / \gamma} Q^{1+\gamma \gamma}
$$

TC : Costos totales

a : Constante

$\gamma$ : Parámetro de la elasticidad

$Q$ : Cantidad ofrecida

Esta forma funcional permite una estimación conveniente parámetro de la elasticidad precio de la oferta. La tienra, el capital y los costos de trabajo están implícitos en Q. La curva de oferta para los cafetaleros es la curva de costos marginales.

$$
M C=\frac{\partial T C}{\partial Q}=(1 / a)^{1 / \gamma} Q^{1 / \gamma}
$$

La función de oferta está representada en la siguiente ecuación:

$$
\begin{aligned}
& P=(1 / a)^{1 / \gamma} Q^{1 / r} \\
& Q=a P^{r}
\end{aligned}
$$

Siendo $P$ y $Q$ la variable explicativa y dependiente respectivamente, resta estimar la elasticidad y la constante. Una ecuación estimable es obtenida tomando logaritmos de la anterior ecuación.

$$
\ln Q=\ln a+\gamma \ln P
$$

b) Un modelo econométrico de la oferta con expectatlvas y ajuste parclal de la producción

El modelo básico para explicar las variaciones en la producción de café es

$$
\ln Q^{\circ}=\ln a+\rho \ln P^{\circ}+\varepsilon
$$

El precio esperado incluye el impuesto a la exportación. El proceso por medio del cual las expectativas se forman depende del comportamiento del precio en anos anteriores. La expansión en la producción viene de dos fuentes. La primera es el incremento en los árboles sembrados. La segunda viene del mejoramiento de las condiciones agr/colas de los árboles existentes. 
Al incrementar el área sembrada, el producto se ajusta con una respuesta diferida debido al ciclo botánico. ${ }^{12}$ Toma al menos cuatro anos para obtener los primeros frutos y los árboles siguen produciendo hasta los 80 anos.

Un periodo más corto de expansión de la producción es posible cuidando mejor la siembra existente. El combate a enfermedades como la roya del café y el cuido de la plantación puede incrementar la producción en un corto período. El precio recibido en antos previos hace que los cafetaleros compren fertilizantes y trabajo adicional para que la producción se incremente con menos rezago.

Hay una respuesta rezagada de la producción después de que la decisión de invertir en el café ha sido tomada. Una respuesta racional es invertir dependiendo de los precios en los años previos. Un rezago pequeno parece el resultado más posible para explicar las variaciones en la producción. La guerra civil minó las motivaciones de los productores para sembrar más árboles.

Ensayamos diferentes tipos de expectativas: desde expectativas ingenuas hasta formas más complicadas. Las diferentes expectativas fueron tratadas en nuestra forma final de estimación. El modelo más significativo con respecto a las variaciones en la producción es aquel que considera que las expectativas de los productores son formadas con el promedio de los dos años anteriores. En la figura 4 los precios han sido graficados considerando las expectativas igual al precio en ese año. La producción y los precios al productor no muestran una clara relación. Nótese la diferencia en la figura 5 en donde los precios se han obtenido mediante el siguiente esquema de expectativas:

$$
P^{e}=\left(P_{t-1}+P_{t-2}\right) / 2
$$

Considerando P como la elasticidad de la oferta en el largo plazo y basados en el modelo de Nerlove (1958), en el cual la producción cambia con respecto al período anterior por una fracción de la diferencia entre el nivel de equilibrio en el largo plazo y su valor actual, tenemos unạ ecuación en primeras diferencias.

$$
\ln Q_{1}-\ln Q_{1-1}=\theta\left(\ln Q^{\circ}-\ln Q_{1-1}\right)
$$

Resolviendo para In $Q$, y substituyendo, se obtiene la siguiente ecuación:

$$
\ln Q_{1}=\theta \ln a+(1-\theta) \ln Q_{1-1}+\theta y \ln P^{\bullet}+E
$$


Teta es el coeficiente de ajuste de la producción en el largo plazo; la elasticidad en el corto plazo es capturada por oy, y en el largo plazo por $\gamma$.

Los resultados de la regresión se muestran a continuación:

$$
\begin{aligned}
& \ln Q_{1}=\underset{(2.76)}{3.27}+\underset{(3.14)}{.46} \ln Q_{1-1}+.18 \ln P^{\circ} \\
& F=13.31) \\
& F \quad D W=2.40 \quad R^{2}=.63
\end{aligned}
$$

De este resultado se infiere que las elasticidades en el corto y largo plazo son 0.18 y 0.33 , respectivamente.

\section{La carga exceslva y el impuesto a la exportación de café}

La carga excesiva del impuesto se ha graficado en la figura 6. La curva con pendiente creciente representa la curva de oferta en el largo plazo. El impuesto reduce el precio recibido por los productores. A este precio los cafetaleros disminuyen la producción. La magnitud de la contracción depende de la sensibilidad de la oferta a los cambios en los precios. El excedente del productor disminuye $A+B$, el gobierno recauda una cantidad igual al rectángulo $A .{ }^{13} \mathrm{El}$ área dada por $B$ no es apropiada por nadie y representa la carga excesiva del impuesto.

Trabajamos con el supuesto de que El Salvador es una nación pequena en el mercado internacional del café; su producción representa menos del $5 \%$ en el mercado mundial, aunque es el cuarto productor de otros suaves. Sobre este supuesto toma el precio como dado, enfrentando una curva de demanda horizontal. El supuesto de pequena nación se mantiene aun con la suspensión del sistema de cuotas, porque El Salvador, a diferencia de Brasil o Colombia, no tiene impacto en el precio mundial del café, y su producción puede ser substituida por la de otros productores.

El impacto del impuesto es la reducción del precio recibido por los productores. Dado que el costo de los factores no se altera, al menos en este modelo, la curva de oferta se mantiene fija. Hay un movimiento simplemente a to largo de la curva de oferta. La implicación es que la tecnología no cambia por la disminución en el precio recibido por los productores, al menos en nuestro esquema de equilibrio parcial.

En esta estructura, el cálculo de la carga excesiva consiste en la estimación del parámetro de la curva de oferta. Una vez que el parámetro de la elasticidad es encontrado, la carga excesiva se obtiene 
integrando el área relevante bajo la curva y substrayéndola del rectángulo formado por la suma de dicha área y la carga excesiva.

Todos los parámetros son identificables. Una vez encontrados la elasticidad y otros parámetros, la carga excesiva (área B en la figura 6) es dada por la siguiente fórmula.

$$
C E=P_{w}\left(Q_{0}-Q_{1}\right)-\int_{\S Q_{1}}^{9 Q_{0}}(1 / a)^{1 /} Q^{1 / \gamma} d Q
$$

La solución de esta integral conduce a la siguiente fórmula:

$$
C E=P_{w}\left(Q_{0}-Q_{1}\right)-(\gamma / 1+\gamma)(1 / a)^{1 / \gamma}\left(Q^{1+\gamma \gamma_{0}}-Q^{1+\gamma \eta_{1}}\right.
$$

Dadas las estimaciones del modelo de produccion, la Tabla 3 presenta la carga excesiva del impuesto a la exportación de café de 1969 a 1987. Podemos notar de que con excepción de 1986, en donde la carga excesiva y los ingresos fiscales totales tuvieron una relación de $7.26 \%$, en otros anos la carga excesiva es menos de $5 \%$. El promedio para todo el periodo es $3.7 \%$.

El análisis de eficiencia demuestra que el impuesto a la exportación no tiene altos efectos distorsionadores. El hecho de que la oferta es inelástica minimiza las distorsiones de este tipo de impuesto.

Esto implica que independientemente del gravamen a la exportación, la producción no se modifica; aunque en el largo plazo afecte los incentivos del sector cafetalero a sembrar más.

\section{Balanza de pagos, tlpo de camblo y el cálculo de la carga excesiva}

En el cálculo de la carga excesiva, estamos comparando una situación de impuesto con una en la que no existe impuesto. Asumiendo que la balanza de pagos está en equilibrio, la eliminación del impuesto produce una expansión en la producción de café (incrementando el volumen de exportaciones). El crecimiento de las exportaciones de café ocasiona un superávit en la balanza de pagos, incrementándose de esta manera el nivel de reservas. Para mantener el equilibrio, el nivel de precios o el tipo de cambio (asumiendo un tipo de cambio flotante), o una combinación de ambos, debe cambiar. En el caso en que sea el tipo de cambio el que se ajuste, éste se tiene que apreciar para 
equilibrar la balanza de pagos. Mientras tanto las importaciones como las exportaciones responden a un nuevo nivel de tipo de cambio. El equilibrio final representa, en el caso del café, un precio al productor menor que el precio recibido en el caso de no ajuste en la balanza de pagos. De esta manera, disminuye la "carga excesiva" del impuesto a la exportación.

A continuación se presenta un modelo simple de la cuenta corriente de la balanza de pagos. Se asume que no hay sector monetario y que el nivel de precios no cambia. El tipo de cambio es la variable que se ajusta para traer el equilibrio.

Las exportaciones de café están representadas por la oferta de café anteriormente calculada. ${ }^{14}$

$$
\begin{aligned}
& \text { In } Q=\gamma_{1}+\gamma_{2} \ln P q+\varepsilon \\
& P q=E P w(1-\gamma)
\end{aligned}
$$

E : Tipo de cambio

Pw: Precio Mundial del Café

La oferta de otras exportaciones es calculada en una forma similar, asumiendo que la oferta de exportaciones en el ano actual es una fracción de su nivel en el largo plazo.

$$
\begin{aligned}
& \ln X=B_{1}+B_{2} \ln P X+\varepsilon \\
& \ln X=\delta_{1}+\delta_{2} \ln P X+\delta_{3} \ln X 1+\varepsilon
\end{aligned}
$$

$P x=E P x$

Px : Precio Mundial de otras Exportaciones

Considerando la demanda de importaciones como una función del precio de las importaciones y del producto interno bruto, tenemos: ${ }^{15}$

$$
\begin{aligned}
& \text { In } M=k_{1}-k_{2} \ln P m+k_{3} \ln y+\varepsilon \\
& P m=E P M
\end{aligned}
$$

$y$ : Producto interno bruto

$P_{M}$ : Precio Mundial de las Importaciones

La balanza es dada por

$$
B=E P X X+E P W Q-E P m M
$$


Después de estimar las equaciones propuestas, la simulación de la situación de no impuesto se obtiene resolviendo el sistema de equaciones compuesto por la ecuación de la balanza de pagos y el siguiente sub-sistema de ecuaciones no lineales:

$$
\begin{aligned}
& Q=\gamma_{1}[E P w(1-\gamma)]^{2} \\
& X=B_{1}(E P x)^{\theta_{2}} \\
& M=k_{1}\left[y^{k^{3}} /(E P m)^{k^{2}}\right]
\end{aligned}
$$

El sistema tiene cuatro variables endógenas y cuatro ecuaciones. La balanza de pagos está en equilibrio en la situación de Impuestos. Removiendo el impuesto, tenemos que el nivel de las variables y el tipo de cambio se altera para mantener el equilibrio.

La estimación del parámetro, produce el siguiente resultado.

$$
\ln \mathrm{Q}=6.01+.33 \ln \mathrm{Pq}
$$

Para la estimación de otras exportaciones, el resto de exportaciones diferentes al café son agregadas. Un índice de precios conteniendo el precio mundial del algodón, café y camarones fue utilizado como un aproximado del precio de otras exportaciones. ${ }^{16}$

$$
\begin{gathered}
\ln X_{1}=\underset{(.7)}{2.75}+\underset{(1.88)}{.4 \ln X_{t-1}}+\underset{(2.79)}{.44} \ln P_{x} \\
D W=1.57 \quad R^{2}=.71
\end{gathered}
$$

De esto obtenernos,

$$
\ln X=4.91+.71 \ln P X
$$

Después de corregir por autocorrelación del primer orden, la ecuación de las importaciones viene dada por: ${ }^{17}$

$$
\begin{aligned}
& \ln M_{t}=9.34-.59 \ln P m t+.93 \ln y_{1} \\
& t \quad(14.3) \quad(-4.6) \quad \text { (4.72) } \\
& D W=1.97 \quad R^{2}=8.3
\end{aligned}
$$


El sistema de ecuaciones simultáneas es resuelto sujeto a la restricción de equilibrio en la balanza de pagos durante la situación de impuesto, y después removiendo el impuesto. El sistema fue resuelto por el método de Newton. El ajuste del tipo de cambio y la carga excesiva son presentados en la Tabla 4. La carga excesiva más alta fue en 1986, representando $3.86 \%$ de la recaudación; el promedio para todo el periodo es $2.2 \%$.

A manera de conclusión podemos sehalar, que el tener un tipo de cambio adecuado, aumenta la eficiencia del impuesto a la exportación, al reducir la carga excesiva.

\section{Concluslones generales}

El sector agricola, y particularmente, el cafetalero ha sido afectado adversamente por diferentes políticas. Además del impuesto a la exportación, el tipo de cambio sobrevaluado, la protección del sector industrial y otros factores, influyen en crear un sesgo en contra del café. En términos de equidad social podria tener algún fundamento el descansar el crecimiento de otros sectores en el rubro del café. Pero analizando las distorsiones en los precios relativos entre sectores, la agricultura y el sector caficultor específicamente estaría subsidiando otros rubros de la estructura económica en El Salvador.

Sin embargo, en lo que al impuesto a la exportación de café se refeire, éste no parece ser tan distorsionador como algunos han sugerido. Esto se debe a la baja elasticidad precio de la producción del grano, de manera que la reducción del precio recibido por el productor a raiz del gravamen a la exportación no causa una alta disminución de la producción. Con todo, el impuesto a la exportación continua acompanado de una carga excesiva.

Consideramos haber probado que el mantener un tipo de cambio adecuado reduce la carga excesiva del impuesto.

No obstante estas consideraciones, los incentivos de los cafetaleros para incrementar la producción pueden verse afectados por estas distorsiones.

No creemos tener una forma definitiva para sefalar la deseabilidad del impuesto a la exportación. En términos teóricos, el impuesto causa distorsiones, aunque hemos verificado empiricamente que no son extremas. Las ventajas en la factibilidad administrativa inclinan la balanza a preferir este tipo de gravamen.

Podrian sefalarse tres puntos que tienen implicaciones de política económica: 
- La reducción de la tasa marginal del impuesto no representa necesariamente un aumento en la producción de café, debido a la baja elasticidad precio de la oferta.

- Mientras no se encuentre otra modalidad de impuesto que tenga factibilidad administrativa, el gravamen a la exportación continúa siendo una fórmula adecuada de recaudación de impuesto.

- La economía en general podría beneficiarse si se evita el sesgo contra el café y el sector agrícola en general, adoptando un tipo de cambio más realista y limitando la protección excesiva al sector industrial.

\section{NOTAS}

1. Ver Lerner y Corden (1974)

2. Para el impuesto óptimo a la exportación ver Rumasset y Setbounsargn (1988) para el caso del arroz, Sánchez Ugarte (1987) para el enfoque de la oferta, Newbery (1987) para el caso del cacao, Repetto (1973) para el del yute en Pakistán. Una buena reseña del impuesto a la exportación aunque no actualizada se puede encontrar en Good, Lent y Ojha (1966).

3. Los ingresos de los cafetaleros eran declarados en el impuesto sobre la renta. Existlan problemas de evasión. El cambio de impuesto (de ingresos a la exportación), era parte de un paquete de medidas económicas orientadas a la industrialización de la economla y en el caso de esta medida fiscal, a la reducción de los costos administrativos.

4. El tratado internacional del caf́e es un acuerdo entre consumidores y productores con el objetivo de alcanzar equilibrio entre la oferta y demanda internacional para obtener estabilidad en los precios.

5. Ver la Tabla 1 para la cuota permitida a El Salvador para el perlodo 1980 1986.

6. El impacto de los altos precios en la expansión de los árboles sembrados fue visible; en 1979 nuevos árboles dieron el primer fruto.

7. La existencia del mercado negro comenzó en 1961, cuando las transacciones en oro y moneda extranjeras fueron declaradas ilegales. De 1982 a 1986 el gobierno estableció un mercado paralelo. Pero las divisas provenientes del café fueron canalizadas a través del cambio oficial. En 1986 una devaluación unificó los dos mercados al cambio oficial de 5 colones por dólar.

8. Ver Corden (1978) y Krueger (1984) para una explicación del sesgo en contra del comercio producido por los aranceles.

9. Para el cálculo de los precios relativos, tomamos el deflactor como precio real de la comodidad. Los precios relativos son obtenidos mediante la división del deflactor del numerario por el respectivo deflactor de otros sectores. 
10. Los precios relativos del café están condicionados por el precio mundial del café, con la excepción del boom en el precio en 1977 y 1987, denotan una tendencia decreciente.

11. Ver Askari y Cummings (1977) y Adams y Behrman (1982) para una revisión de las estimaciones de las respuestas de la oferta en la agricultura.

12. Para las características botánicas del café ver Wellman (1961).

13. Asumiendo que toda la producción es exportada.

14. Recordemos que el total de producción se exporta.

15. Un supuesto restrictivo es la exogeneidad del producto interno bruto.

16. En la revista del Banco Central, se puede obtener estadisticas sobre el total de las exportaciones kilogramos. Pesos subjetivos fueron asignados a los precios en el índice de precios dependiendo de su participación en las exportaciones, y fueron deflactados por el indice de precios al consumidor.

17. El precio de las importaciones fue obtenido dividiendo el valor deflactado de las importaciones por la cantidad total en kilogramos.

\section{BIBUOGRAFIA}

- Adams, Behrman (1982), Commodity exports and economic development. Lexington Books.

- Asjarum Cummings (1976), ASurvey of The Econometric Evidence. Praeger Publishers.

- Corden, W(1974), Trade Policy and Economic Welfare. Oxtord University Press.

- Diamond and Mirrlees (1971), Optimal Taxation and Public Production. Part I American Economic Review vol 61 \#1, part II, American Economic Review vol 61 \#3. 
TABLA 2

ESTIMACION DE LA ELASTICIDAD DE LA OFERTA DE CAFE

\begin{tabular}{|c|c|c|c|c|c|}
\hline Pals & Periodo & Autor & Ano & $\begin{array}{l}\text { Conto } \\
\text { plazo }\end{array}$ & $\begin{array}{l}\text { Largo } \\
\text { plazo }\end{array}$ \\
\hline Brazil & $1930-55$ & Arak & 1967 & 2.02 & \multirow[b]{2}{*}{$\begin{array}{l}.54 \\
.28\end{array}$} \\
\hline $\begin{array}{l}\text { Sao Paulo } \\
\text { Minas Gerais } \\
\text { Esplritu Santo } \\
\text { Colombia }\end{array}$ & & & & $\begin{array}{l}.08 \\
.20 \\
.47 \\
.84 \\
63\end{array}$ & \\
\hline Colombia & $\begin{array}{l}194 /-65 \\
1952-65\end{array}$ & Bateman & 1968 & $\begin{array}{l}.63 \\
.64\end{array}$ & \multirow{2}{*}{$\begin{array}{l}1.33 \\
1.07\end{array}$} \\
\hline $\begin{array}{l}\text { Uganda } \\
\text { Kenya }\end{array}$ & $\begin{array}{l}1900-38 \\
1946-64\end{array}$ & $\begin{array}{l}\text { Frederick } \\
\text { Maitha } \\
\text { Ford }\end{array}$ & $\begin{array}{l}1969 \\
1970 \\
1971\end{array}$ & & \\
\hline $\begin{array}{l}\text { Jamaica } \\
\text { Africa }\end{array}$ & $\begin{array}{l}1953-68 \\
1947-73\end{array}$ & $\begin{array}{l}\text { Williams } \\
\text { De Vries } \\
\text { Berhman }\end{array}$ & $\begin{array}{l}1972 \\
1975 \\
1977\end{array}$ & $\begin{array}{l}.7 \\
.12\end{array}$ & \multirow{2}{*}{$\begin{array}{l}.44 \\
.3 \\
.53\end{array}$} \\
\hline desarrollo & & $\begin{array}{l}\text { Askari and } \\
\text { Cummings }\end{array}$ & 1977 & .37 & \\
\hline
\end{tabular}


TABLA 3

CARGA EXCESIVA DEL IMPUESTO A LA EXPORTACION

\begin{tabular}{|r|r|r|r|}
\hline Año & Recaudacion & Carga excesiva & Carga excosiva \\
\hline 1969 & 117458 & 3395 & 2.89 \\
1970 & 147358 & 3589 & 2.43 \\
1971 & 122356 & 3251 & 2.65 \\
1972 & 113444 & 2399 & 2.11 \\
1973 & 197410 & 7203 & 3.64 \\
1974 & 138181 & 4645 & 3.36 \\
1975 & 140450 & 5875 & 4.18 \\
1976 & 340435 & 13190 & 3.87 \\
1977 & 811324 & 41403 & 5.10 \\
1978 & 418542 & 21257 & 5.07 \\
1979 & 318127 & 12956 & 4.07 \\
1980 & 203307 & 6585 & 3.23 \\
1981 & 150099 & 6234 & 4.15 \\
1982 & 138487 & 5138 & 3.71 \\
1983 & 86732 & 2844 & 3.27 \\
1984 & 122290 & 4860 & 3.97 \\
1985 & 74887 & 2190 & 2.93 \\
1986 & 460707 & 33447 & 7.26 \\
1987 & 103821 & 4093 & 3.94 \\
\hline
\end{tabular}


TABLA 4

AJUSTE EN EL TIPO DE CAMBIO Y CARGA EXCESIVA

\begin{tabular}{|c|c|c|c|c|c|}
\hline Año & $\begin{array}{c}\text { Tipo } \\
\text { de camblo }\end{array}$ & $\begin{array}{l}\text { Tpo de } \\
\text { camblo }\end{array}$ & $\begin{array}{l}\text { Recau- } \\
\text { daclón }\end{array}$ & $\begin{array}{c}\text { Carga } \\
\text { exceslva }\end{array}$ & Carga oxcos \\
\hline 1969 & 2.5 & 2.438 & 114545 & 2396 & 2.09 \\
\hline 1970 & 2.5 & 2.448 & 114293 & 2553 & 1.76 \\
\hline 1971 & 2.5 & 2.446 & 119713 & 2348 & 1.96 \\
\hline 1972 & 2.5 & 2.468 & 119713 & 1902 & 1.69 \\
\hline 1973 & 2.5 & 2.438 & 192514 & 5450 & 2.83 \\
\hline 1974 & 2.5 & 2.440 & 134865 & 3472 & 2.57 \\
\hline 1975 & 2.5 & 2.394 & 134495 & 3777 & 2.80 \\
\hline 1976 & 2.5 & 2.408 & 327907 & 8791 & 2.68 \\
\hline 1977 & 2.5 & 2.357 & 764916 & 24774 & 3.23 \\
\hline 1978 & 2.5 & 2.065 & 345715 & 1890 & 0.54 \\
\hline 1979 & 2.5 & 2.384 & 303366 & 7842 & 2.58 \\
\hline 1980 & 2.5 & 2.435 & 197208 & 4482 & 2.27 \\
\hline 1981 & 2.5 & 2.371 & 142354 & 3573 & 2.51 \\
\hline 1982 & 2.5 & 2.387 & 132227 & 3011 & 2.27 \\
\hline 1983 & 2.5 & 2.405 & 83436 & 1733 & 2.07 \\
\hline 1984 & 2.5 & 2.368 & 115833 & 2674 & 2.30 \\
\hline 1985 & 2.5 & 2.382 & 71352 & 1067 & 1.49 \\
\hline 1986 & 5.0 & 4.485 & 413254 & 15973 & 3.86 \\
\hline 1987 & 5.0 & 4.493 & 93293 & 1014 & 1.08 \\
\hline
\end{tabular}


Figura 1

\section{El Salvador}

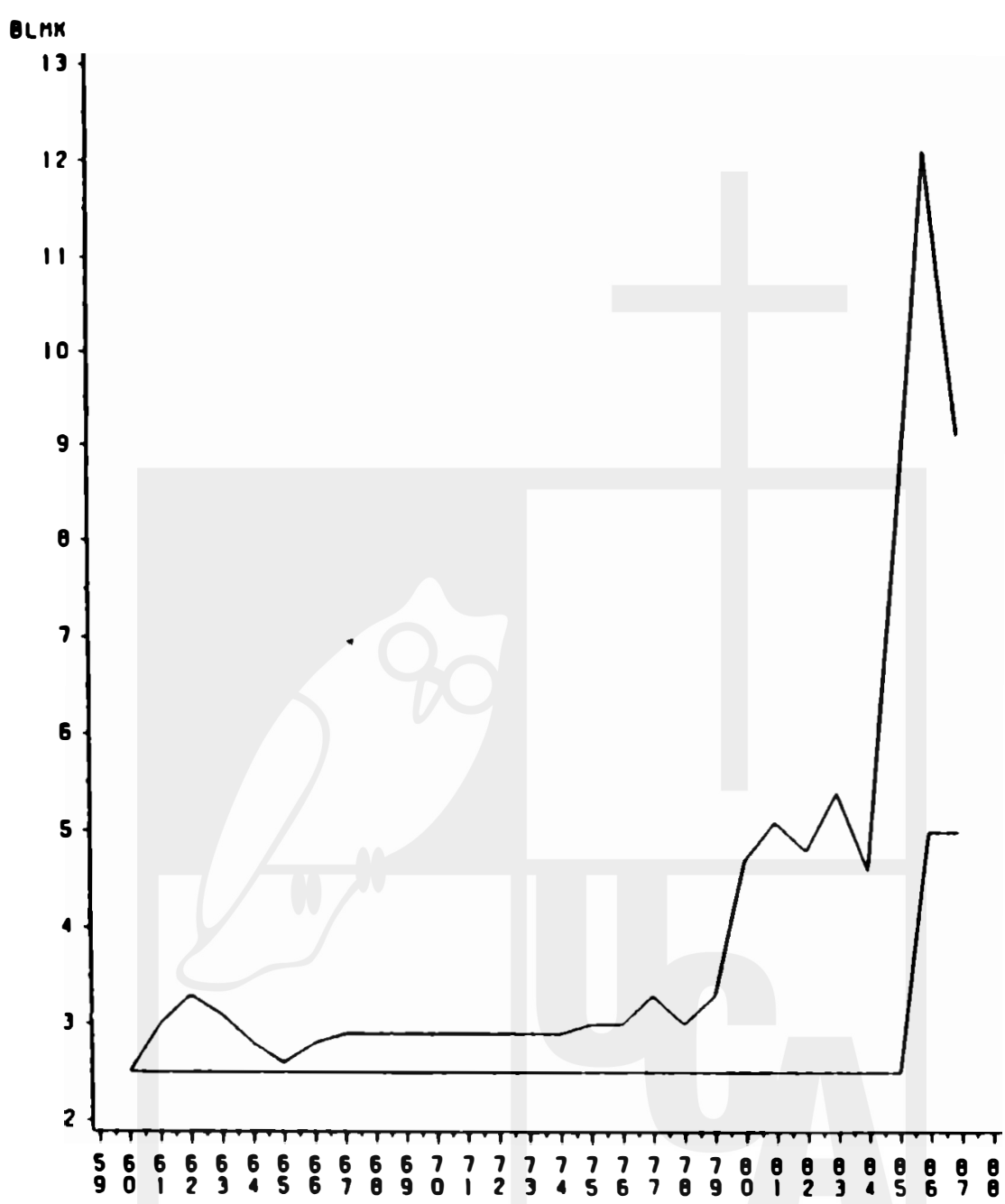

\section{Años}

Tasas oficiales y de mercado negro. 
Figura 2

Precios relativos en el sector agrícola

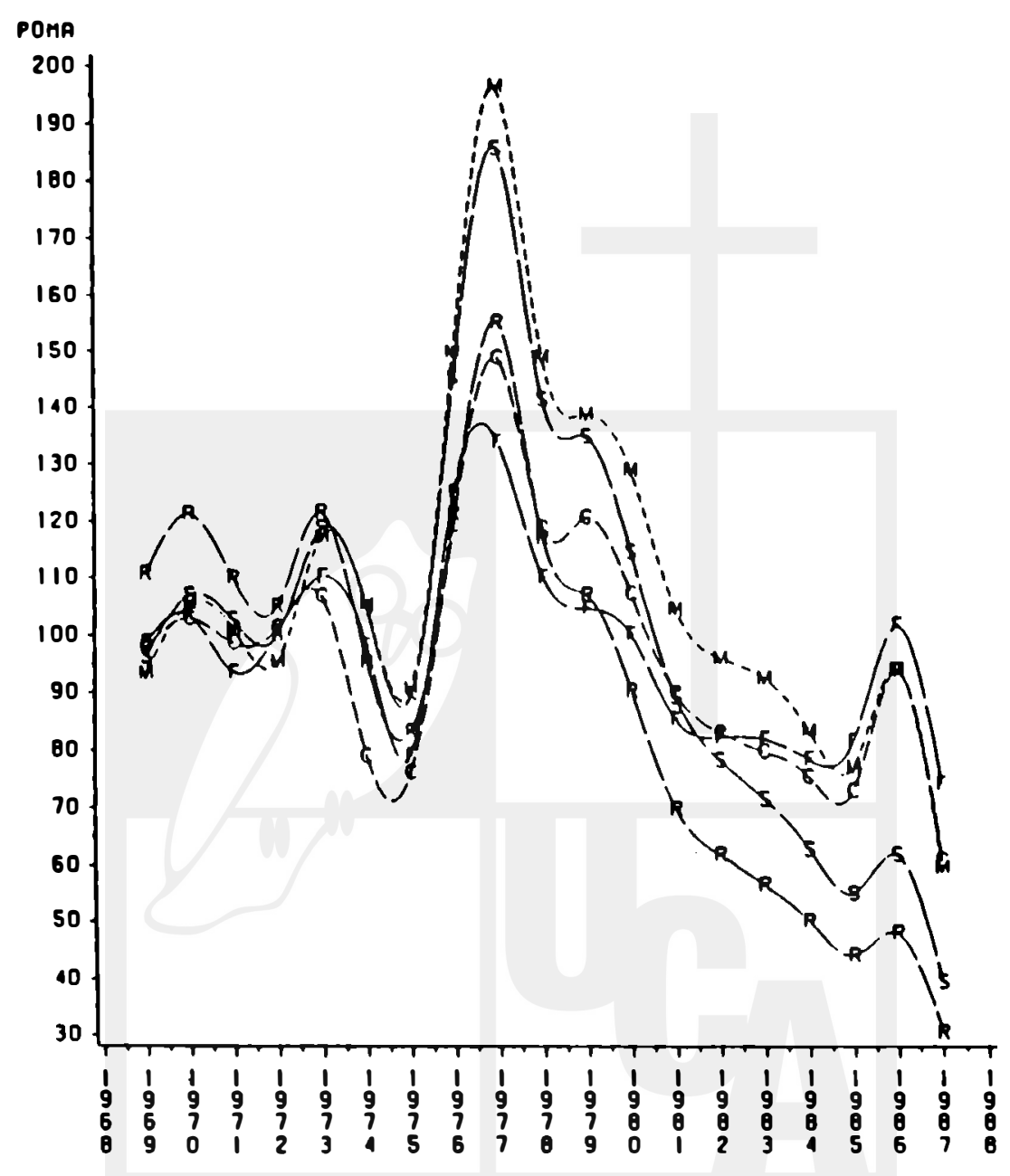

Años

Fuente: Revista del Banco Central de Reserva. 
Figura 3

Precios relativos en el sector cafetalero

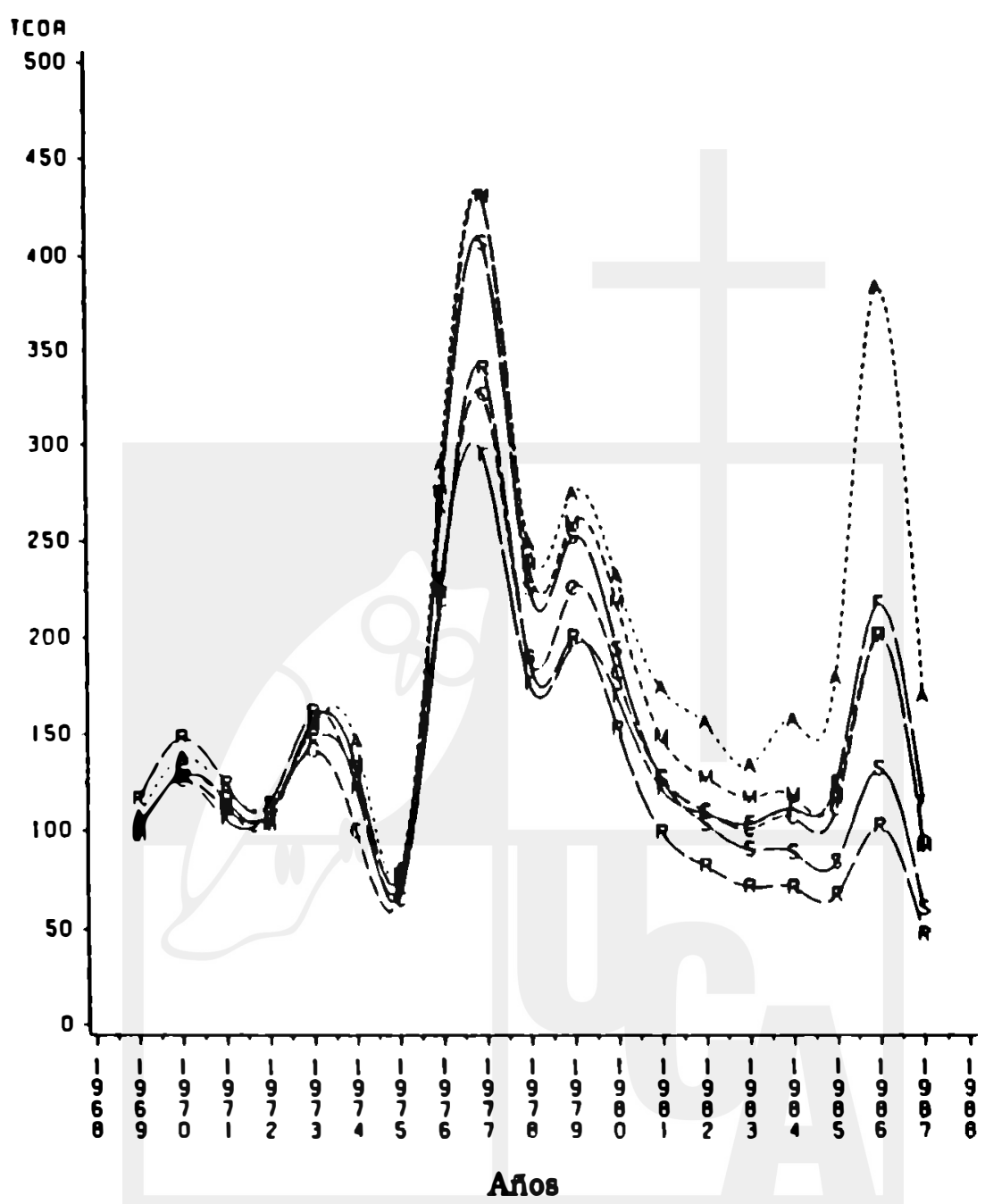

Fuente: Revista del Banco Central de Reserva. 
Figura 4

Producción y precio recibido por los cafetaleros

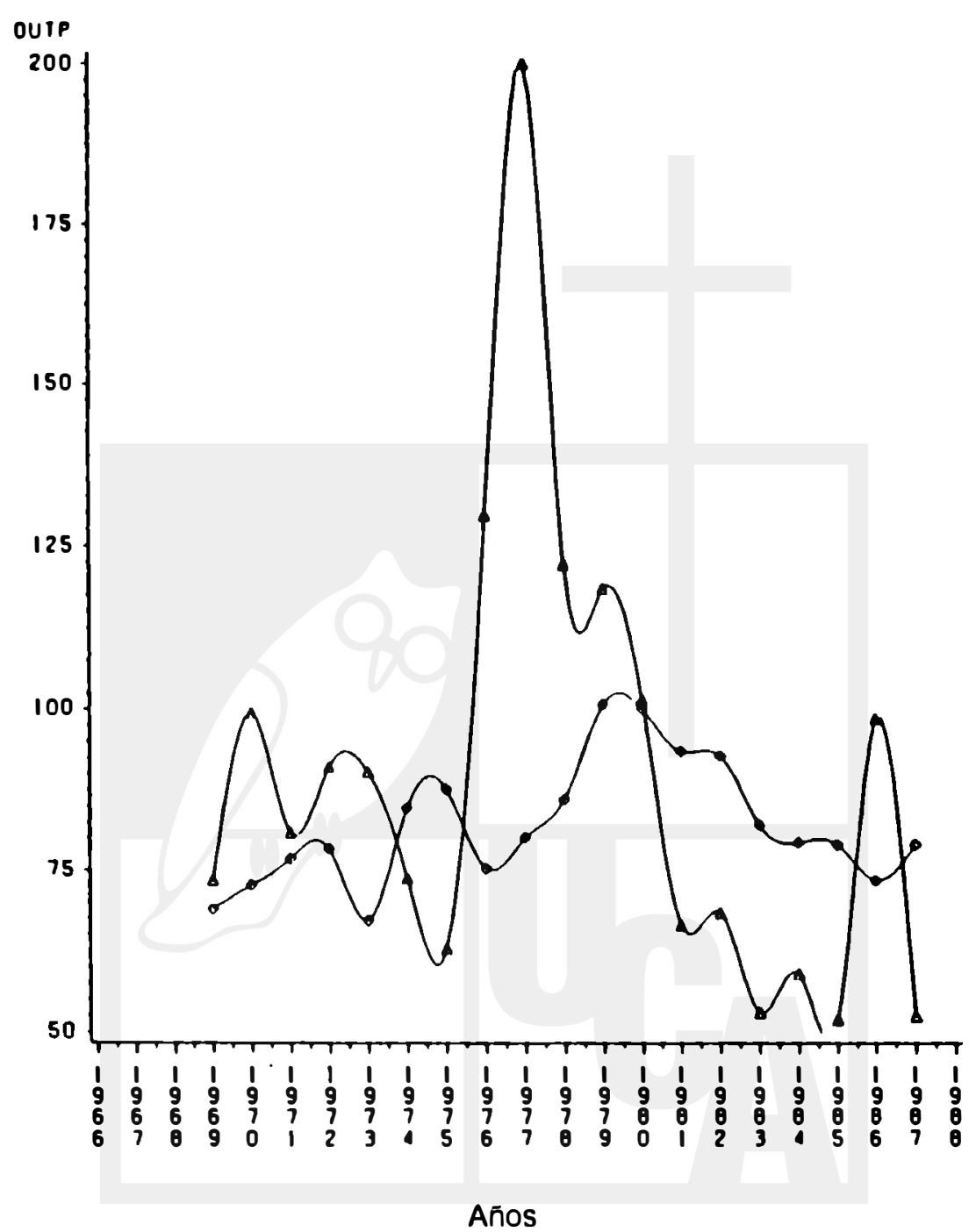

Precios en el mismo año.

Fuente: Revista del Banco Central de Reserva. 
Figura 5

Producción y precio recibido por los cafetaleros

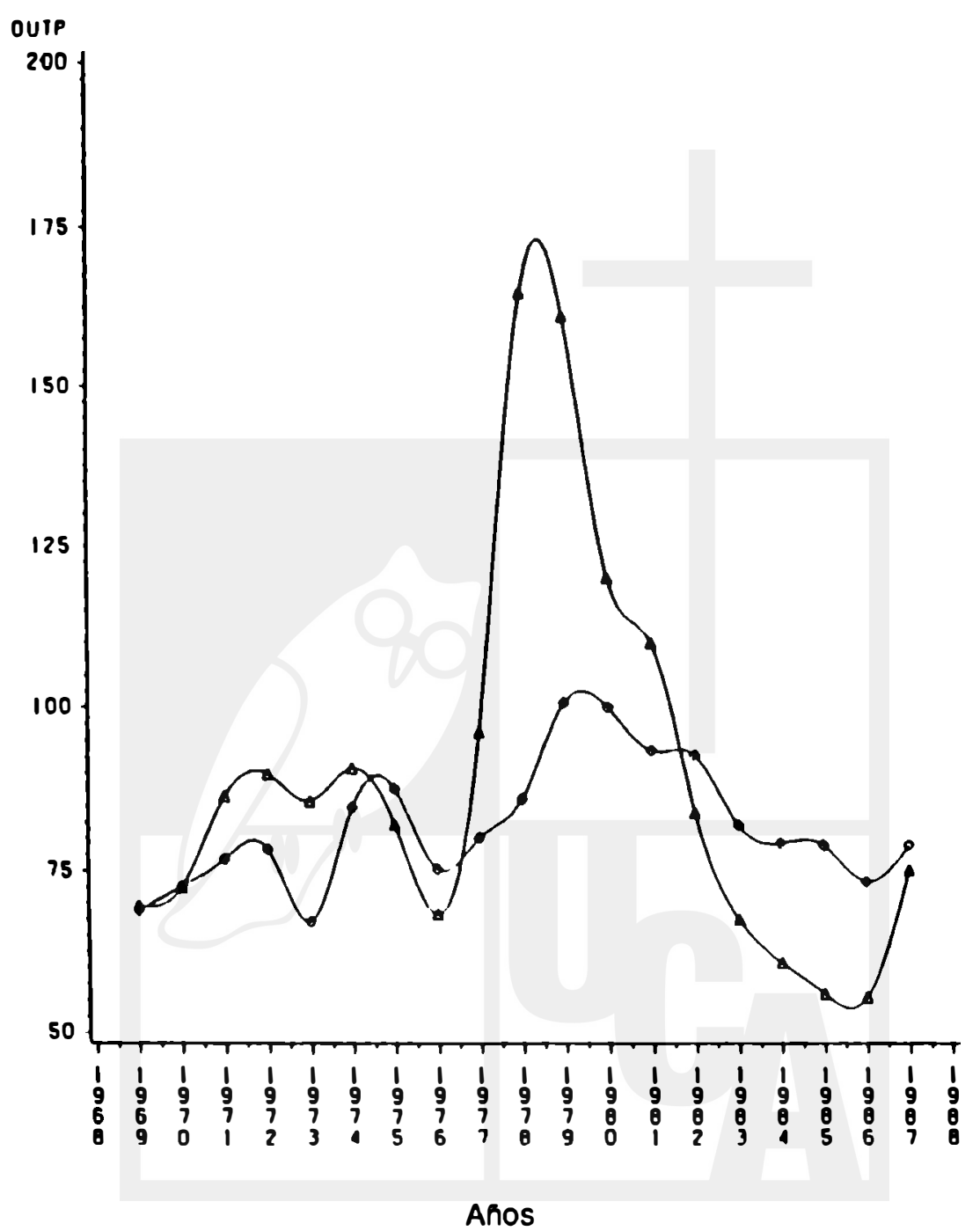

Promedio de los años anteriores.

Fuente: Revista del Banco Central de Reserva. 
Figura 6

\section{IMPUESTO A LA EXPORTACION}

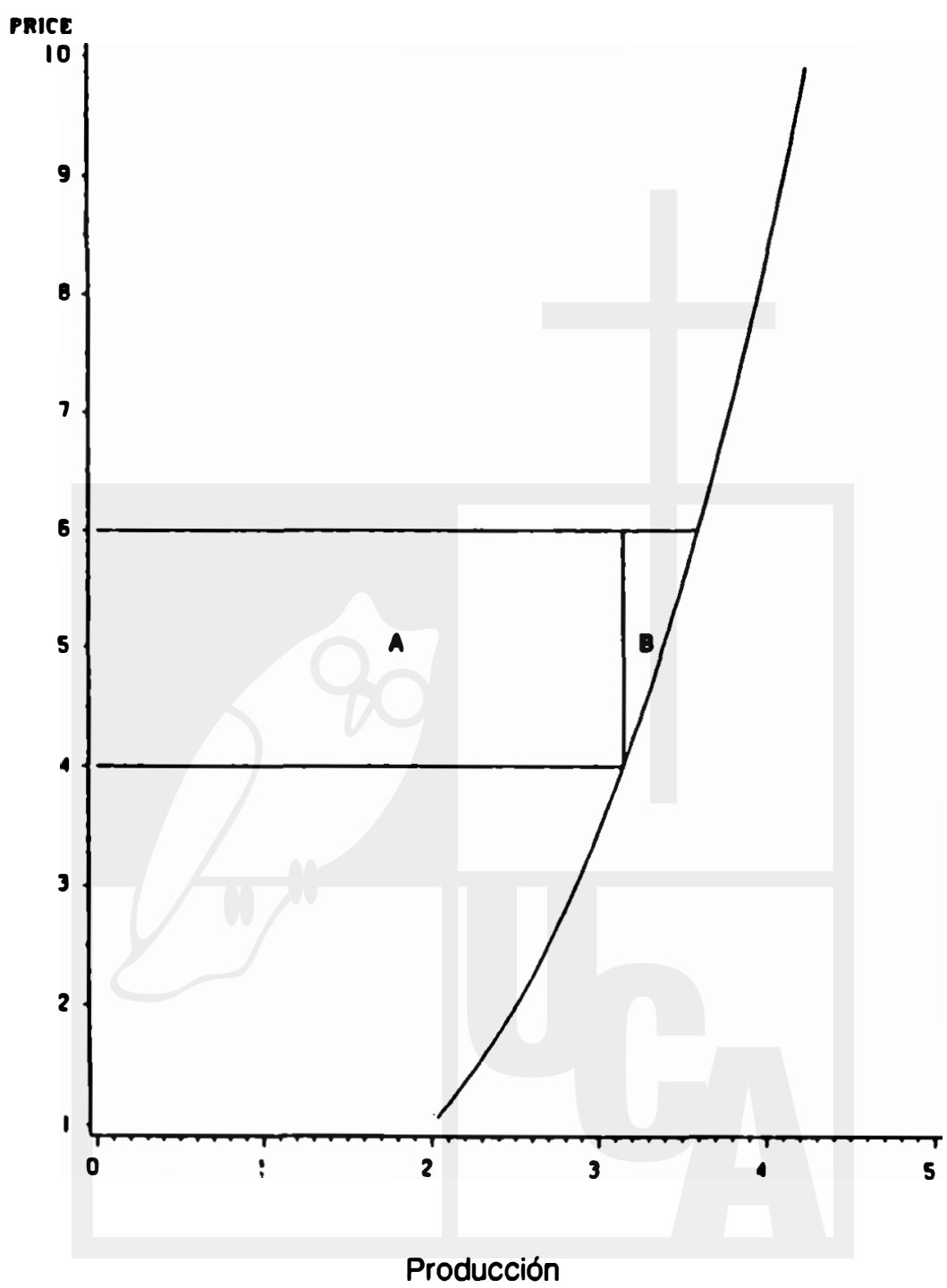

Carga excesiva o peso muerto 